\title{
Strong Lensing by High-Redshift Red-Sequence Selected Galaxy Clusters
}

\author{
Michael D. Gladders ${ }^{1}$ \\ ${ }^{1}$ Carnegie Observatories, 813 Santa Barbara St., Pasadena CA, 91101, USA
}

\begin{abstract}
The statistics of giant arc formation by galaxy clusters has long held promise as a cosmological tool. Progress in this field relies upon advances in modeling, and in observational samples. We introduce several new lens samples from the Red-Sequence Cluster Survey (RCS1) which provide a significant increase in the total sample of clusters with arcs, while adding several new observational puzzles. The most significant of these observations is that the redshift distribution of clusters which form arcs is unexpectedly skewed to higher redshifts. We also discuss upcoming samples from the ongoing RCS-2 survey and other planned surveys; preliminary results from RCS-2 support this unexpected redshift distribution and deepen the disagreement between theory and observations.
\end{abstract}

\section{Introduction}

The formation of arcs due to strong gravitational lensing by galaxy clusters is dependent on essentially three factors: 1) the properties of the clusters (lenses), including the evolving cluster mass and correlation functions, 2) the properties of background objects (sources) and 3) the cosmology and its effects on lensing geometry. Most elements of 1) and 2) are either observable (such as source and lens redshift distributions) or amenable to modeling (such as the detailed mass distributions in lenses). Some aspects of the lens population are intimately connected to the cosmology, since the cosmological model has a significant effect on the growth of structure on these scales. Elucidation of cosmological parameters through analysis of cluster lensing thus becomes a tricky business, with the cosmology playing a role in both the geometry and in the gravitational (and perhaps baryonic) physics of cluster formation. That there is much room for improvement in our understanding of the latter, even from a (relatively speaking) simple n-body modeling perspective, is evidenced by several papers presented at this meeting (Dalal 2004; Meneghetti 2004) and their order-of-magnitude changes in lensing cross sections from previous works.

Theoretical issues aside, there are also significant observational limitations to using cluster lensing as a cosmological tool. Galaxy clusters are rare objects - and massive clusters even more so. At typical ground-based imaging depths and spatial resolutions, even most of the most massive clusters do not show any significant evidence for strong lensing; strong lensing by clusters is an extremely rare phenomenon, and current samples are correspondingly small. Moreover, the apparent incidence of strong lensing by clusters is a strong function of observational constraints (such as wavelength, depth, and spatial resolution); compare, for example, the few arcs in early ground-based imaging of Abell 1689 (e.g., Tyson, Valdes \& Wenk 1990) to the plethora of arc systems in the spectacular deep ACS imaging presented at this meeting (Sharon et al. 2004). Establishing the necessary bridge between cosmological expectations and observational realities is non-trivial, and made more complex when one invokes multiple and often mutually exclusive arc samples in order to boost statistics. 
There are further difficulties in the comparison between theory and observations when considering arc statistics. The most obvious difficulty when discussing arc counts is that the lenses are drawn from a steep mass distribution which is often observationally probed at only the most massive end. The observed and predicted arc counts are thus sensitive to the actual mass limit (in the case of observations) or presumed (deduced) mass limit (in the case of theoretical predictions). While most arcs so far discovered are formed by massive clusters, there are also clear examples of arc formation by clusters of rather pedestrian masses (e.g., RCS0348.8-1017 [Gladders et al. 2003]; CL 2244-02 [Ota, Mitsuda \& Fukazawa 1998]). In principle then, one must fully understand the detailed mass limitations of any given observational survey in order to make a reliable comparison to theoretical predictions. The mass limits in a given survey are usually not simple since the relationship between mass and relevant survey observables is significantly scattered. Moreover, the secondary parameters which contribute to the selection functions in surveys (such as cluster concentration) often also affect the lensing cross sections. One way of surmounting this mass-limit problem is to examine differential properties rather than global counts, such as redshift distributions and multiplicity ratios of lenses (e.g., Gladders et al. 2003), or the location of tangential versus radial arcs (e.g., Williams, Navarro, \& Bartelmann 1999). Unfortunately the effective use of such distributions generally requires better statistics than the Poisson-limited samples which exist currently. However, new cluster lens samples are beginning to appear, and we expect a resurgence in this field in the coming decade. Below we discuss the RCS-1 results, some tantalizing hints from the RCS-2 and SDSS surveys, and provide some expectations for future surveys.

\section{The RCS-1 Strong Lensing Cluster Sample}

The new sample of clusters with arcs from the RCS-1 (Gladders et al. 2003; Gladders \& Yee 2004) provides some limited measure of the redshift distribution of clusters lenses out to a redshift of approximately $z=1$, and shows that many of the clusters with arcs tend to form multiple arc systems due to multiple background sources at widely separated redshifts. The RCS-1 results also confirm that the expected number of strong lensing systems, at least to the moderate depths of RCS-like imaging, is about one per ten square degrees. Four examples of the lensing systems from this sample are shown in Figure 1. With the addition of one system reported elsewhere in the literature (Gladders, Yee \& Ellingson 2002) there are 5 strong lensing systems in the RCS-1 taken directly from the survey data, and an additional 3 from secondary follow-up imaging of clusters at $\mathrm{z} \sim 1$.

The large proportion of clusters showing multiple arcs seems now to be well in hand from a theoretical perspective, with recent papers finding multiplicity ratios similar to the observations (Ho \& White 2004). Moreover the density of arcs on the sky seen in various observations (in addition to the RCS-1, see for example Zaritsky \& Gonzales 2003) is now well matched by the most recent modeling. However the redshift distribution of the RCS1 lenses is at this point not well matched by the current modeling (e.g., Dalal, Holder \& Hennawi 2004). However, a sample of 5 objects is likely insufficient to be a cause of great concern; again the modest sizes of current samples limit the utility of these type of comparisons.

\section{Hints From RCS-2 and the SDSS}

The follow-on survey to RCS-1 is the RCS-2 survey, a $~ 1000$ square degree $g r z$ survey to a depth comparable to RCS-1, now underway at CFHT. A quick analysis of the first 


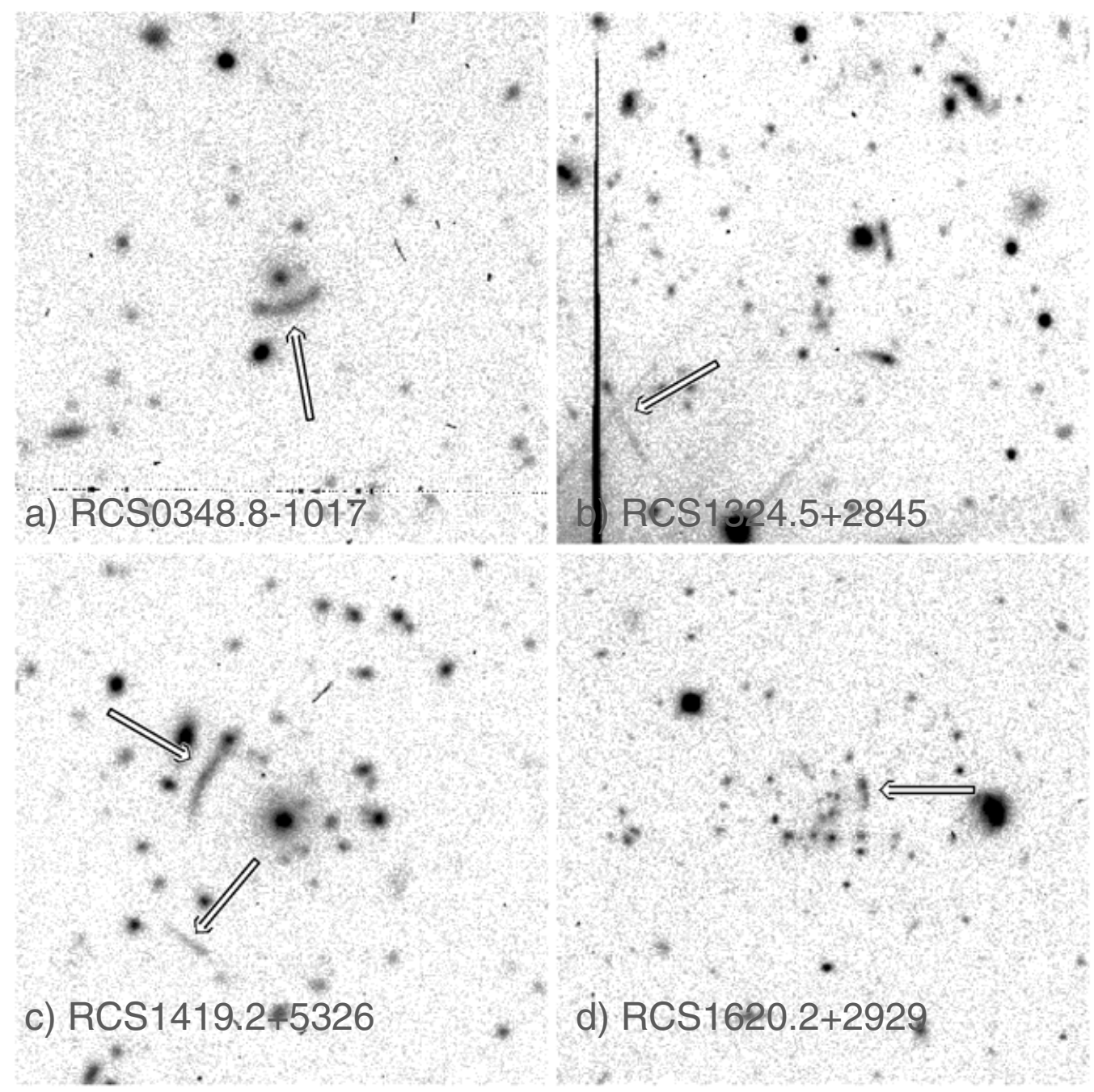

Figure 1. R-band images of strong lenses from the primary sample of the RCS-1, from Gladders et al. (2003).

$\sim 12$ square degrees of data from a portion of the first semester's observations for this survey yields one further cluster with at least one arc. This strong lens candidate is shown in Figure 2. Notably the cluster appears to be at a photometric redshift of $\sim 0.6$ - again at odds with the expectation from theory.

Analysis of both the primary Sloan Digital Sky Survey† (SDSS) imaging, as well as the co-added data from the deep southern strip of the SDSS is ongoing by several groups

$\dagger$ Funding for the creation and distribution of the SDSS Archive has been provided by the Alfred P. Sloan Foundation, the Participating Institutions, the National Aeronautics and Space Administration, the National Science Foundation, the U.S. Department of Energy, the Japanese Monbukagakusho, and the Max Planck Society. The SDSS Web site is http://www.sdss.org/. The SDSS is managed by the Astrophysical Research Consortium (ARC) for the Participating Institutions. The Participating Institutions are The University of Chicago, Fermilab, the Institute for Advanced Study, the Japan Participation Group, The Johns Hopkins University, the Korean Scientist Group, Los Alamos National Laboratory, the Max-Planck-Institute for Astronomy (MPIA), the Max-Planck-Institute for Astrophysics (MPA), New Mexico State University, 


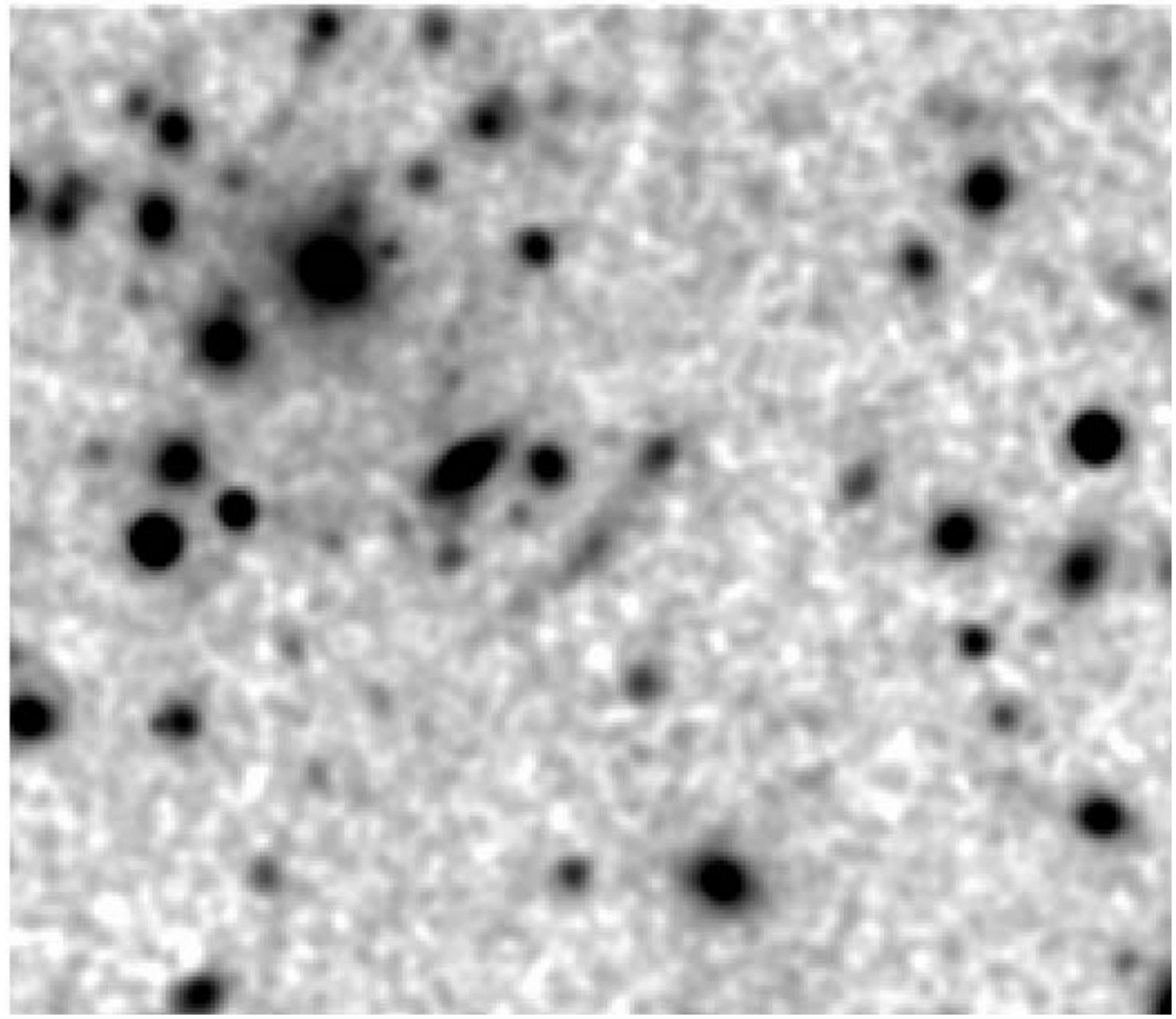

Figure 2. The first candidate giant arc from the RCS-2 survey. The image is a smoothed version of the survey $g$-band image with a greyscale range chosen the emphasize the arc candidate in the center of the image. The cluster, at $\mathrm{z} \sim 0.6$ is to the top left.

(private communications, H. Lin, J. Hennawi) and a number of strong lens candidates have been found. The deeper data are of particular interest since the cluster selection function for these data should be similar to the RCS-1; in particular massive clusters well above redshift 0.5 are accessible in images of this depth. The first strong lens candidate in these data with follow-up imaging is shown in Figure 3. Again, the cluster is at $z \sim 0.7$, an unexpectedly high redshift

\section{Future Expectations}

Preliminary indications from the surveys described above are that the redshift distribution of cluster strong lenses is strongly at odds with current theoretical expectations. The disagreement is strong but not overwhelmingly significant, given the limited number of clusters. This will change shortly.

The RCS-1 results, and the preliminary analysis of the first RCS-2 data confirm previous work (e.g., Zaritsky \& Gonzales and references therein) which indicates that the density of arcs on the sky to these depths is about one per 10-20 square degrees. By this

University of Pittsburgh, Princeton University, the United States Naval Observatory, and the University of Washington. 


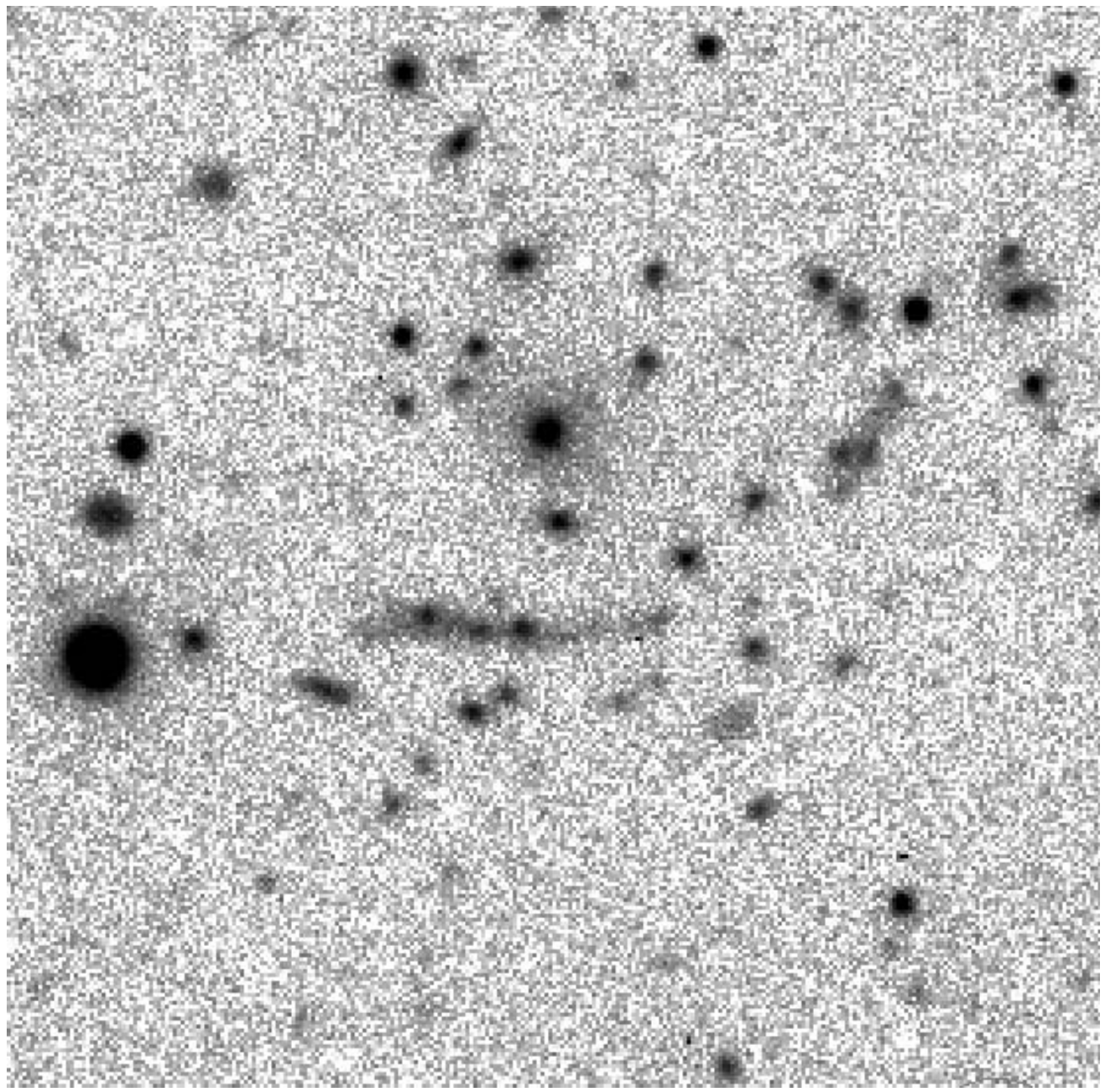

Figure 3. The first candidate giant arc from the SDSS southern stripe (found by H. Lampeitl, $\mathrm{H}$. Lin, and J. Annis). The image is a composite $B V R$ image taken with the IMACS imaging spectrograph on the Magellan I telescope image. The greyscale range has been chosen to emphasize the arc candidate near the center of the image.

metric the RCS-2 survey should find 50-100 such clusters and the Dark Energy Survey (DES, 5000 square degreest) should provide samples of hundreds of uniformly selected strong lensing clusters. Notably the DES is a deeper survey than either the RCS-1 or RCS-2 (by 0.3 to more than 1 magnitude depending on the filter) and given the steepness of the background source counts with magnitude it is not unreasonable to expect $\sim 1000$ clusters with arcs in the DES. Other surveys to various depths will also contribute significantly. Strong lensing clusters samples of this scale will require significant resources to compile the extensive follow-up data (particularly spectroscopy) needed to take full advantage of these objects. The discovery space for cluster strong lenses is about to expand dramatically; the bottleneck for understanding these rare objects will shortly be large telescope time, rather than the lack of samples which has historically been the limiting factor.

$\dagger$ http://cosmology.astro.uiuc.edu/DES 


\section{Acknowledgements}

M.D.G. gratefully acknowledges the the collaborative support of various members of the RCS team, particularly David Gilbank, Henk Hoekstra, and Howard Yee. Without their support none of the work described herein would be possible. I am also indebted to Huan Lin for detailed comments and the various SDSS collaborators working on arcs in the SDSS data for their collaborative spirit, and permission to use the image in Figure 3 of a yet unpublished SDSS arc discovery.

\section{References}

Dalal, N., Holder, G. \& Hennawi, J.F. 2004, ApJ, 609, 50

Gladders, M.D., \& Yee, H.K.C. 2004, ApJS, in press

Gladders, M.D., Hoekstra, H., Yee, H.K.C., Hall, P.B., \& Barrientos, L.F., \& Hall, P.B. 2003, ApJ, 593, 48

Gladders, M.D., Yee, H.K.C., \& Ellingson, E. 2002, AJ, 123, 1

o, S., \& White, M. ApJ, submitted, astro-ph0408245

Ota, N., Mitsuda, K, \& Fukazawa, Y. 1998, ApJ, 495, 170

Sharon, K. et al. 2004, this proceedings

Tyson, J.A., Valdes, F., \& Wenk, R.A. 1990, ApJL, 349, 1

Williams, L.L.R., Navarro, J.F., \& Bartelmann, M. 1999, ApJ, 527, 535

Zaritsky, D. \& Gonzales, A.H. 2003, ApJ, 584, 691 